\title{
A IMAGÉTICA KINETICA E MENTAL EM PRATICANTES DE DESPORTOS COLECTIVOS EINDIVIDUAIS
}

\author{
MENTAL AND KINETIC IMAGERY AMONG ATHLETES OF \\ COLLECTIVEANDINDIVIDUALSPORTS
}

\author{
José Vasconcelos RAPOSO' \\ Goreti COSTA \\ Isabel Mouráo CARVALHAL \\ Universidade de Trás-os-Montes e Alto Douro
}

\begin{abstract}
RESUMO
Opropósito deste estudo foi avaliar e comparar os valores de imagética mental e cinética em jovens praticantes de vários niveis competitivos de desportos colectivos e individuais, de dois niveis de escolaridade, com diferentes anos de prática e que nunca foram submetidos a uma programa de preparação psicológica para a competição nem a qualquer tipo de teste psicológico.

A amostra foi constituida por 366 individuos, 152 do sexo feminino e 214 do masculino. A média das idades foi 20.29 anos. Os sujeitos aceites para o estudo foram subdivididos em grupos de acordo com as variáveis independentes definidas para este estudo. Assim, para o nível de escolaridade integraram o grupo UTAD, 205 indivíduos (50 fem. e 155 masc.) e o grupo 12A com 161 sujeitos (102 fem. e 59 masc.). Quando organizados por tipo de desporto constamos que 143 eram praticantes de modalidades individuais (31 fem. e 112 masc.) e 117(44 fem. e 73 masc.) de colectivas.

Os resultados da comparação por sexos evidenciaram diferenças estatisticamente significativas na componente imagética mental. Foram encontradas diferenças entre os diferentes grupos da variável anos de prática.

Os valores obtidos tendem a reforçar algumas das conclusões já publicadas e discutidos na literatura desta área do saber. A forma como interpretamos os resultados recorrem a argumentos diferentes daqueles habitualmente constatados na literatura da especíalidade.
\end{abstract}

\footnotetext{
(1) José Vasconcelos-Raposo é Professor Associado em Psicologia na Universidade de Trás-os-Montes e Alto Douro. Goreti Costa é licenciada em Educação Física e Desporto e Isabel Mourão Carvalhal é Professora Auxiliar na Universidade de Trás-os-Montes e Alto Douro. A correspondência relativa a este artigo deverá ser endereçada para o primeiro autor e para o seguinte endereço: UTAD- Extensão de Miranda do Douro. R. D. Díniz. Miranda do Douro, 5210 - Portugal. jraposo@utad.pt
} 
Palavras-chaves: Imagética mental, imagética cinética, visualização, iniciados.

\begin{abstract}
The purpose of the present study was to compare levels of mental and kinetic practice among young athletes involved in collective and individual sports. The independent variables were: level of school attendance, years of sports practice. Having been subjected to a program of mental training was used as thee inclusion criteria in the sampling procedure.

The sample consisted of 366 individuals (152 females and 214 males). The average age was 20.29 years. These subjects were divided into groups according to the independent variables of this study. For the school levei there were 205 individuais in Ihe group "UT" (50 famels and 155 males) and 161 subjects in Ihe group "12A" (102 remates and 59 males). When organized according to Ihe type of sport, 117athletes practiced a collective sport (44 remates and 73 males) and Ihe individual sports group consisted of 143 subjects (31 remates and 112 males).

The results showed that there were statistical significant differences between Ihe sexes for mental imagery. There were also significant differences between Ihe groups according to Ihe years of practice.

These results tend to confirm some of Ihe previous research published in this scientific area, however Ihe arguments that we use to explain some Ihe values obtained differ from Ihe known literature.
\end{abstract}

Key-words: Mental imagetics; Kinetic imagetics; visualization; beguns

Consciente ou inconscientemente todos sonhamos acordados e conseguimos imaginar coisas, recordações. A imagética é a habilidade de nos vermos a nós próprios a desempenhar tarefas, evocando pensamentos e imagens, com um fim. Esta habilidade consiste em recuperar a informação armazenada na memória (através de todo o tipo de experiências) e remodelá-la através dos processos cognitivos.

A imagética é, cada vez mais, uma das componentes mais importantes na preparação psicológica dos atletas e treinadores para a competição (O'Halloran \& Gauvin, 1994). A visualização, como área de estudo, tem merecido a atenção dos cientistas de várias disciplinas. Mais recentemente esse interesse foi retomado em diferentes domínios do saber, entre os quais a filosofia da ciência, ciências cognitivas, neurociências e psicologia do desporto, para mencionar apenas algumas. A habilidade humana para elaborar imagens mentais tem desempenhado um papel importante no desenvolvimento de vários corpos teóricos que visaram explicar o funcionamento da mente, quer em tempos remotos como presentemente.

O interesse académico sobre a "imagem mental" tem sofrido algumas oscilações. Numa primeira fase, foi tema central na psicologia, mas como desenvolvimento e popularização do comportamentalismo tendeu a ser afastada das discussões académicas, para re-emergir nas últimas décadas.

Apesar de existir na literatura material suficiente para argumentar que a imagética é importante para a melhoria do rendimento desportivo, continuamos sem ter explicações 
suficientemente elucidativas sobre a forma como esta funciona. Daí a necessidade de aprofundarmos o nosso conhecimento nesta área do saber da psicologia aplicada ao desporto.

Para tornar mais perceptível o interesse que este tema tem despertado ao longo dos séculos, assim como as razões que nos levam atribuir-lhe uma acentuada relevância no desenvolvimento de corpos teóricos, julgamos necessário fazer uma breve descrição sobre a forma como foi tratado ao longo dos tempo.

O estudo científico da visualização foi iniciado com Fechner em 1860 e posteriormente retomado por Galton em 1880. Outros investigadores, para além dos já acima mencionados, no início deste século, deram o seu contributo para o avanço do conhecimento nesta área, entre os que mais se destacaram foram Stetson (1808), Colvin (1908), Betts (1909), Schaub (1911), e Angell (1912), que demonstraram existir diferenças nas capacidades dos indivíduos em imaginar através dos vários órgãos dos sentidos. Com o trabalho de Schaub (1911) e de Fernald (1912), emergiram as primeiras propostas para a existência de vários tipos de imagética, sendo deste logo identificada a diferença entre a perspectiva cinestésica e "visual" (mental).

Entre os primeiros filósofos, como Aristóteles, já se poderiam identificar algumas preocupações com esta capacidade. Este pensador, por exemplo, defendeu que o "pensamento era impossível sem imagens" (Kosslyn, 1980). Os filósofos falaram da psicologia em termos da mente, a qual pensaram que recebia informação que era mediada e direccionada para a forma de comportamentos. Segundo os seus argumentos, a mente pode contemplar algo que está ausente, servindo-se de representações de objectos ou de acontecimentos. Quando algo já não está em frente do sujeito, este, para pensar nele (imaginá-lo), terá de o representar mentalmente.
O problema colocou-se sobre a forma que os objectos tomavam como representações na mente. Certamente que não eram palavras, porque estas estão arbitralmente relacionadas com algo que as significa. Por exemplo, quando se aprende a palavra gato associa-se a palavra ao felino de quatro patas e que mia, mas não existe nada de especial sobre os sons que usamos para o referir. As imagens, ao contrário das palavras, não são arbitrariamente relacionadas com algo que representam. Ter uma imagem é como ter o objecto, mas sem o ter naturalmente presente. Reconhecemos o objecto em imagem da mesma forma que $o$ reconheceríamos se o estivéssemos a ver. Assim, para alguns, as imagens, como conceito, ofereceram uma solução para o problema de como é que a mente representa objectos e acontecimentos. Conseqüentemente, alguns filósofos aceitaram o pensamento como sendo constituído por sequências de imagens. Esta ideia cedo começou a perder o seu apelo, entre outras razões, porque reconheceram que as pessoas podem pensar sobre "classes" de objectos, e não apenas em alguns em particular. Por outras palavras, um indivíduo pode pensar sobre triângulos em geral sem no entanto estar a especificar se são só triângulos equiláteros, isósceles, e assim sucessivamente. Ainda que as imagens sejam utilizadas para representar informação, elas não são a única forma de representação dos objectos e conceitos que usamos no nosso dia-a-dia.

Contudo, a imagem mental como tema de estudo tornou-se popular entre os primeiros psicólogos, em parte, graças à forma e ao significado que atribuíram à introspecção como meio para aprender sobre a mente humana. Wilhelm Wundt fundou o primeiro laboratório de psicologia em Leipzig, Alemanha, em 1879, e a introspecção foi uma das metodologias a que recorreram. A aproximação de Wundt à psicologia foi feita com o intuito de construir algo como uma "tabela periódica da percepção interna" na qual os elementos a considerar 
eram as imagens. As regras de combinações de imagens visaram ditar todos os possíveis tipos de experiência acessíveis a uma pessoa. Os progressos feitos conduziram a um debate empolgado e rico, na viragem do sec. $X X$, em especial no que referia à defesa do "pensamento imagético".

Alguns seguidores de Wundt, em particular Titchener, examinaram as suas introspecções e ao reflectirem sobre elas discordaram uns dos outros. Titchener foi mesmo ao ponto de afirmar que possuía imagens mesmo para ideias muito abstractas, como "significandos". O problema levantado com o chamado pensamento imagético, não foi o facto da divergência de ideias, mas sim o problema de não existir uma forma credível e aceite pela comunidade científica para resolvêlo. Dois grupos relataram dois tipos de introspecção: como seria possível demonstrar qual dos dois estaria correcto?

Perante este dilema, John B. Watson, que leccionou por uns tempos na Universidade de John Hopkins, encontrou-se num impasse e assumiu uma resolução radical, argumentando que eventos mentais, de qualquer tipo, não era um assunto apropriado para a psicologia. Com esta posição a psicologia entrou num caminho diferente daquele que até então tinha assumido, retomando-se uma perspectiva mais mecanicista e cedo o comportamentalismo seguiu esta mesma direcção, mas com alguma oscilação. Watson considerou as conversas sobre imagética como isso mesmo: "conversas". Ele defendeu que, se queremos estudar o "pensamento", deveremos moderar subtilmente a actividade em vários músculos da garganta, porque o pensamento, segundo Watson, era simplesmente falar consigo mesmo. Durante o período de influência primordial dos comportamentalistas, desde 1913 até finais dos anos 50, os estudos de todos os eventos e/ou actividades mentais foram evitados e a imagética foi virtualmente ignorada.
O pêndulo começou o seu baloiço para trás nos finais dos anos 50 , e actualmente a imagética é objecto de muita investigação. Por que razão se deu o retrocesso? Acreditamos que existem três razões: A primeira, foi que a fragilidade do comportamentalismo tornou-se evidente e, de certo modo, porque não foi clara a forma a explicação dos comportamentalistas sobre a percepção ou o uso da linguagem, a sua aquisição e relacionamento com o pensamento. A segunda, das razões, prendeuse com a ruptura evidente do impasse metodológico sobre a controvérsia do "pensamento imagético". Assim sendo, novas metodologias foram inventadas, as quais se tornaram facilitadoras para os investigadores, no sentido de ser possível estudar objectivamente a actividade e os eventos mentais. Estas metodologias permitiram a exteriorização desses acontecimentos, e em vez do estudo da mente propriamente dita, tornou-se possível quantificar as variáveis que moderava mas consequências observáveis dos processos internos. A última razão para o despontar do interesse no estudo da imagética centrou-se nos desenvolvimentos que a investigação na área da linguística e inteligência artificial tiveram. $O$ trabalho de Noam Chomsky demonstrou a necessidade de ordenar as representações não observáveis, para assim compreender-se melhor como as pessoas utilizam a linguagem. Para este estudioso, se os computadores podem ser programados para solucionar problemas, também a mente poderá operacionalizar-se como um programa computadorizado. Esta analogia foi bastante importante, na medida em que, entendendo como funcionam os computadores, e pensando na mente da mesma forma, foi possível afastar a aura de misticismo que anteriormente rodeava as conversas sobre os eventos e actividades mentais.

Consciente ou inconscientemente, todos sonhamosacordadose conseguimos visualizar 
coisas, recordações. A visualização é a habilidade de nos vermos a nós próprios a desempenhar tarefas, evocando pensamentos e imagens, com um fim. Esta habilidade consiste em recuperar a informação armazenada na memória (através das várias modalidades sensoriais) e remodelando-a através dos processos cognitivos.

A eficácia da visualização está intimamente relacionada com a memória e as recordações, por esta razão pode ajudar no desenvolvimento de capacidades, graças à forma como as sensações estão associadas às recordações e imagens. Podemos usar os sentimentos para aumentar as imagens visuais e usar as imagens visuais para controlar os sentimentos, atitudes, emoções e comportamentos.

Kosslyn (1988) definiu a imagética ou visualização "... como um estado do cérebro semelhante aos que têm lugar durante a percepção, mas que ocorre na ausência de estimulação imediata; tais eventos são normalmente acompanhados de uma experiência consciente como é o ver com os olhos da mente e ouvir com ouvidos da mente, etc." (p.1621). Este estudioso realça, ainda, o facto de existirem alguns aspectos comuns entre a visualização e a percepção no que se refere aos mecanismos de funcionamento do organismo. Em parte este saber advém do aprofundamento dos conhecimentos emergentes com o avanço da tecnologia que permite estudar aspectos da fisiologia da visão que até então não foram possíveis.

A imaginação é uma das capacidades mais negligenciadas e pouco desenvolvida no ser humano. Através da visualização podemos recriar experiências tidas no passado e, detalhadamente, transportá-las para o presente e assim poder reconstruí-las, ajustando-as ao estado emocional que se deseja.

Desde sempre o uso de imagens mentais foi utilizado pelos atletas. Recentemente, várias técnicas de visualização mental foram aplicadas ao desporto, para elaborar respostas alternativas de pensamentos, sensações e atitudes. Muitos atletas descobriram que 0 uso dessas imagens serve para aperfeiçoar, ou melhor, valorizar a prestação. No entanto, constatou-se que apesar de um número elevado de atletas recorrer a esta técnica não conseguem os efeitos que desejam. Em parte, estes efeitos nefastos resultam do facto de que nunca foram devidamente sensibilizados para desenvolver todo o potencial desta habilidade psíquica, que para produzir melhoria qualitativa das prestações, requer o seu treino de uma forma sistemática.

A visualização como técnica, serve vários propósitos:

- ajuda o atleta nos processos de aprendizagem e melhoria das técnicas (tarefas motoras);

- desenvolve e aperfeiçoa estratégias de competição através do ensaio mental das situações de competição (treino mental);

- proporciona a aquisição das habilidades psicológicas para lidar com as situações que tendem a ocorrer nos períodos que antecedem as competições, assim como durante estas.

Com a prática da visualização, o atleta pode ensaiar todas as opções e situações que, de outra maneira, não estariam ao seu alcance na prática real. Assim, tudo pode ser preparado antecipadamente.

As técnicas de visualização (associadas a verbalizações "self-talk"), ajudam o atleta a:

- aumentar a sua autoconfiança;

- acelerar o processo de aprendizagem de uma nova habilidade motora;

- alterar maus hábitos comportamentais ou de execução motora;

- melhorar a consistência de prestação, de duas maneiras: 1- resolução de problemas: nesta situação recorre à 
visualização com o intuito de aumentar a autoconfiança, reduzir o stress competitivo, a tensão física e planear possíveis formas de acção e ajudar na incrementação do despertar fisiológico previamente definido como o desejável para o atleta: 2- processo de se imaginar a si próprio a realizar as tarefas inerentes à competição em que irá participar.

Talvez uma das formas mais eficazes para destacarmos a importância da visualização na preparação dos atletas é fazendo referência às posições públicas que alguns dos melhores atletas do mundo têm tomado. Por exemplo, o famoso jogador de golf Jack Nicklaus defendeu que $50 \%$ do sucesso, na colocação da bola onde pretendeu, resultou da imagem mental que desenvolveu para o efeito. Greg Luganis, vencedor de oito medalhas de ouro nos Jogos Olímpicos de 1984, recorreu sistematicamente à visualização para treinar os saltos para a água e afirmou complementar este treino com música, que tudo indica foi benéfico para o processo de melhoria do "timing" nas sequências motoras envolvidas. A tenista Chris Evert-Loyd assegurou que esta técnica Ihe ajudou a melhorar as suas prestações. Nas palavras da nadadora Maria Carlos Santos nunca é demais visualizar, quanto mais melhor. Vasconcelos-Raposo (1994a) referiu que 21\% dos atletas portugueses de elite que participaram nos Jogos Olímpicos de Barcelona recorreram à visualização para controlar os níveis de activação fisiológica, ensaiar as prestações que tiveram, assim como para melhorar as suas capacidades técnicas.

No mundo do desporto, observamos, frequentemente, que a diferença entre vencedores e vencidos foi apresentada em termos de centímetros, fracções de segundo, uma pequena distracção por parte do atleta, um erro numa das componente técnicas, etc.. Perante este cenário, são cada vez mais os atletas e treinadores que procuram trabalhar a componente mental, de modo a poderem manter-se nos limites que os rigores da competição exigem. Ao longo dos anos, vários estudos constataram que uma percentagem razoável de atletas de elite recorrem à prática da visualização (Vasconcelos-Raposo, 1994A , 1994; Murphy, Jowdy \& Durtschi, 1990). Por exemplo, Vasconcelos-Raposo (1994) estudou vinte e oito (28) atletas olímpicos e constatou que $80 \%$ destes recorreram a esta técnica para lidar com várias das situações que tendem a perturbar-Ihes a concentração durante o período competitivo.

Neste contexto, julgamos ser da maior pertinência estudar a visualização. No estudo realizado por Vasconcelos-Raposo (1994) verificou-se que a visualização foi uma das variáveis que mais diferenciou os atletas portugueses dos vários níveis competitivos, tendo os atletas com melhor nível de rendimento desportivo obtido melhores valores para esta prática. Revisões narrativas da literatura, levadas a cabo por Richardson (1967a;1967b) e Zaichkowsky e Fuchs (1988), Hall, Schmidt, Durand, e Buckolz, 1994) demonstraram a relevância desta componente para o perfil psicológico de prestação. Feltz e Landers (1983), no seu estudo meta-analítico sobre a visualização, concluíram que a prática mental foi superior à ausência de execução motora, $e$ que a primeira, quando associada à segunda, produz efeitos na melhoria da prestação de forma significativamente superior a qualquer uma das duas outras, quando treinadas isoladamente.

De acordo com Gould et al. (1989), a visualização foi a técnica de preparação mental mais utilizada pelos psicólogos do desporto nos Estados Unidos de América. Um outro trabalho, realizado por Jowdy et al. (1989), quantificou essa utilização e concluiu que esta foi usada por $100 \%$ dos psicólogos, $90 \%$ dos atletas e $94 \%$ dos treinadores. Num outro estudo, realizado por (1990) constatou-se que 90\% dos 159 olímpicos estudados e $94 \%$ dos treinadores inquiridos integraram a visualização 
nos seus planos para se prepararem para as competições. Para Perry e Morris (1995), a popularidade desta técnica poderá estar associada ao facto de todos nós, num momento ou outro, sonharmos acordados com as coisas que gostaríamos que acontecessem, ou até mesmo com experiências positivas ocorridas no decurso da nossa vida.

A história da investigação nesta área peca por não apresentar uma descrição detalhada sobre a natureza da prática mental, os seus mecanismos e processos. No entanto, os resultados dos estudos parecem apontar claramente para o facto de a prática mental contribuir para a melhoria do rendimento desportivo, assim como para o desempenho de qualquer outra tarefa, especialmente as de carácter motor.

A evidência empírica sugere que há diferenças entre os atletas em função do rendimento destes. Clark (1960), argumentou que a prática mental talvez fosse mais benéfica para atletas com um nível de habilidade reduzido. Em oposição a esta tese, Start (1962) verificou que foram os atletas com melhor nível de prestação que mais ganharam com a aplicação da técnica da visualização. Perry e Morris (1995), porém, realçaram que foram os indivíduos que melhor construíram imagens mentais e que melhor as controlaram que mais benefícios retiraram do seu uso. Estes autores ainda nos alertaram para o facto de a prática mental poder ser benéfica para aqueles atletas que assumem a responsabilidade das suas acções, nomeadamente no que se refere ao controlo da sua preparação psicológica.

As vantagens da utilização da visualização foram demonstradas empiricamente em vários domínios: na aquisição de habilidades motoras, prestação de mergulho, rendimento em atletismo (Ungerleider \& Golding, 1991), dança (Vaccaro, 1997), programas de controlo da agressividade em jovens (Lennings, 1996), melhoria do rendimento escolar (Cullinan,
1999), preparação dos doentes que se submeterão a intervenções cirúrgicas (Tusek, Church, \& Fazio, 1997), marketing (Bone \& Ellen, 1992), assim como em outras áreas (ver Feltz \& Landers, 1983; Richardson, 1967; Corbin, 1967; Weinberg, 1982; Feltz, Landers \& Becker, 1988; Gordon, Weinberg \& Jackson, 1994).

A utilização da visualização por atletas é algo que está suficientemente documentado (Vasconcelos-Raposo, 1994; Jowdy, Murphy, \& Durtschi, 1989; Hall, Rodgers \& Barr, 1990). De acordo com Wiggins (1984), já no final do século 19, William Anderson, um professor de Educação Física, realizou estudos na área da psicologia do desporto e defendeu a prática da visualização. Em 1916,Washburn argumentou que quando os indivíduos visualizam podem observar os seus movimentos, e que estes se diferenciaram dos movimentos reais pela sua reduzida magnitude. O seu argumento assentou no princípio que quando os indivíduo visualizam ocorre o mesmo tipo de actividade muscular, só que neste processo as sensações são mais modestas. Jacobson, (1930), validou este argumento e esclareceu que quando os indivíduos testados foram mais experientes nos movimentos imaginados as suas sensações e os movimentos observados foram mais acentuados. Mais recentemente, Richardson (1967a; 1967b), com base na análise que fez a vinte e cinco estudos, concluiu que a prática da visualização contribuiu para a melhoria das prestações. Feltz e Landers (1983), com base numa meta-análise de 60 artigos publicados, chegaram à mesma conclusão.

Janssen e Sheikh (1994) argumentaram que a literatura científica existente sugere que a imagética ou visualização é uma método eficaz e eficiente no processo de melhoria de rendimento desportivo dos atletas. Os mesmos autores também referem que esta técnica, quando utilizada inadequadamente pode ter efeitos contrários aos desejados. Daí que seja recomendável a sua aplicação sob a orientação 
de um psicólogo devidamente treinado para $o$ efeito.

No contexto do desporto recorre-se, essencialmente, a dois tipos de visualização: a interna e a externa. A primeira designou-se por visualização kinética e a segunda de visualização mental. De acordo com Hale (1982) e Suinn (1994) a visualização interna é uma aproximação à fenomenologia da vida real, de tal forma que permite à pessoa visualizar e sentir, no seu corpo, as sensações que à priori espera sentir na situação real. $\mathrm{A}$ visualização externa é quando o sujeito se vê na perspectiva de um observador externo. Tal como Suinn (1994) escreve, é como se a pessoa se observasse num filme.

De acordo com Suinn (1994) o valor de cada uma das formas da visualização depende do nível de experiência com que cada um se inicia na prática do treino da visualização. Assim, o objectivo deste trabalho é caracterizar o nível inicial da visualização kinética e mental em jovens não praticantes e praticantes de modalidade colectivas e individuais nunca submetidos a qualquer tipo de preparação mental para a competição.

\section{METODOLOGIA}

Este estudo é do tipo quasi-experimental. Este paradigma é, em alguns aspectos, muito semelhante ao método experimental. Recebe a designação de quasi-experimental na medida em que é impossível, ao investigador, aplicar todos os aspectos do segundo. Para melhor se entender esta classificação é necessário esclarecer as relações existentes entre os tipos de variáveis utilizadas em cada um dos casos.

As variáveis de sujeito são aquelas que não podem ser manipuladas pelo experimentador. Exemplo deste tipo são a idade, o sexo ou a inteligência dos que constituem a amostra a ser estudada. Este aspecto, de acordo com Wood (1977), é da maior importância na medida em que se prende com o tipo de conclusões a que é possível chegar. Por outras palavras, porque não se pode exercer qualquer tipo de controlo sobre as variáveis em causa as conclusões a tirar oferecem uma margem de erro relativamente grande, uma vez que poderão existir outros tipos de explicação para os resultados obtidos. De qualquer forma, o método quasi-experimental oferece como vantagem a possibilidade de estabelecer novas validações externas.

A amostra do presente estudo foi de conveniência e foi constituída por 366 indivíduos, 152 do sexo feminino e 214 do masculino. A média das idades foi 20.29 (. Os sujeitos aceites para o estudo foram subdivididos em grupos de acordo com as variáveis definidas como independentes. Assim, para o nível de escolaridade integraram o grupo UTAD, 205 indivíduos (50 fem. e 155 masc.) e o grupo 12A com 161 sujeitos (102 fem. e 59 masc.). Quando organizados por tipo de desporto constamos que 143 eram praticantes de modalidades individuais (31 fem. e 112 masc.) e relativamente às colectivas o número de praticantes foi 117 (44 fem. e 73 masc.). O número de praticantes por tipo de modalidade, nível de escolaridade e sexo estão definidos no quadro 1 .

\section{Quadro I. Número de praticantes por tipo de modalidade, nivel de escolaridade e sexo.}

\begin{tabular}{|llccc|}
\hline & & Fem. & Masc. & Total \\
\hline Colectivo & UTAD & 23 & 59 & 82 \\
& 12A & 21 & 14 & 35 \\
Individual & UTAD & 27 & 92 & 123 \\
& $12 \mathrm{~A}$ & 4 & 16 & 20 \\
Totais & & 75 & 185 & 260 \\
\hline
\end{tabular}

Quando o grupo foi dividido em função dos anos de prática estes foram organizados nos seguintes grupos: 1 a 5 anos (grp1), 6 a 10 
anos (grp 2) e 11 e mais anos de prática (grp3). Integraram o primeiro grupo $67 \mathrm{em}$ desportos individuais e 62 em colectivos. No segundo grupo 62 nas individuais e 45 nos colectivos e no grupo três a distribuição foi de 14 para as individuais e 10 para as colectivas. O teste de "pearson Chi-square" (367.37: $p=0.000$ ) demonstrou que estes grupos estavam devidamente divididos. A divisão relativamente ao sexo feminino evidenciou: grp1 $=47$, grp2 $=23$ e grp3 $=5$. Para os indivíduos do sexo masculino a divisão foi: grp1= 82; grp2= 84 e grp3= 19 .

\section{Procedimentos e instrumento}

Para os efeitos do presente estudo recorremos ao único instrumento para a língua portuguesa, que conhecemos, traduzido e validado do original de Hall e Pongrac (1983) com a designação de Imagery Movement Questionnaire, que em português recebeu a designação de "Questionário sobre Imagética do Movimento" (Vasconcelos-Raposo \& Costa, 1997).

Este questionário consiste em 18 questões que têm por objectivo mensurar duas variáveis do foro psicológico tidas como de primordial importância para o rendimento desportivo: visualização cinética e mental. A aplicação deste instrumento requer alguns cuidados, nomeadamente no que se refere à familiarização do experimentador com o instrumento e com a prática da visualização. Para cada um dos itens é solicitado ao sujeito que realize uma tarefa e que seguidamente a procure reproduzir cinética e visualmente. Por outras palavras, há sempre uma posição inicial, uma acção motora e uma tarefa mental. A falta de experiência tende a traduzir-se em tempos inadequados para a imagética quer cinética quer mental. Após a tarefa cada indivíduo avalia, numa escala de 1 a 7 , a facilidade / dificuldade que teve em no desempenho da tarefas de representação anotando-as, imediatamente após a sua realização, numa folha que está ao seu lado. Ao valor 1 corresponde o muito fácil e ao 7 muito difícil de representar. Os valores das escalas oscilam entre o 9 e os 63 pontos. Os itens da escala cinética são todos os impares e os pares são os da escala mental.

Os participantes foram reunidos num ginásio para o propósito da realização do teste onde os ruídos ambientais que pudessem interferir na concentração necessária à administração do questionário e realização das tarefas inerentes ao mesmo foram minimizadas $A$ apresentação dos itens foi feita sob o controlo do experimentador que os apresentou de acordo com a ordem em que estes se apresentam no teste. Não se verificaram dificuldades durante esta fase da recolha de dados.

As dificuldades sentidas ao longo do processo deste estudo prendeu-se com a falta de sensibilidade para a investigação científica por parte de alguns educadores, que por falta de formação científica não reconhecem a importância das actividades físicas no desenvolvimento das crianças.

\section{Procedimentos estatísticos}

As técnicas estatísticas utilizadas foram: $\mathrm{t}$-Test, para compararmos dois grupos e a Anova-uma via sempre que comparamos três grupos. Para comparar os praticantes em função do sexo, tipo de modalidade e nível de escolaridade, recorremos à estatística multivariada (GLM: General Linear Model). Para os cálculos de GLM foram introduzidos como factores fixos as variáveis sexo, modalidade e escolaridade, como covariáveis a idade e anos de prática. As variáveis dependentes deste estudo foram: visualização kinética, mental e a de Eu- atleta.

Sempre que o número de indivíduos que integravam cada um dos grupo se apresentou desigual e quando esses grupos tinham menos de 12 indivíduos recorremos à estatística 
não-paramétrica. Para os propósito deste estudo utilizamos o software do SPSS 10.0.

\section{APRESENTAÇÃO DOS DADOS}

A abordagem a esta parte do trabalho é feita através da apresentação dos dados obtidos através das técnicas estatísticas aplicadas. Assim, em função das variáveis independentes tomadas em consideração, começamos por descrever os valores referentes à estatística descritiva.

Diferenças por sexo. A média das idades dos estudantes do sexo feminino da UTAD foi $20,08( \pm 1,82)$ e dos rapazes $21,61( \pm 3,17)$ anos de idade. A média para os sujeitos do sexo feminino do $12 A$ foi $19,02( \pm 1,33)$ anos de idade e a dos rapazes foi $19,17( \pm 1,10)$ anos.

Procuramos saber se existiam diferenças significativas entre os rapazes e raparigas ao nível das duas dimensões da visualização, assim como da autopercepção como atletas. Os resultados evidenciaram que não há diferenças estatisticamente significativas para a variável autopercepção. No entanto, para a dimensão mental verificou-se diferenças significativas ( $\mathrm{t}=20.181, \mathrm{p}=0.030$ ).

\section{Quadro II. Diferenças entre sexos para as variáveis kinésicas, mental e auto percepção.}

\begin{tabular}{|lccccc|}
\hline EUATLETA & $\mathrm{N}$ & $\mathrm{M}$ & $\mathrm{DP}$ & $\mathrm{t}$ & $\mathrm{p}$. \\
\hline Feminino & 75 & 6.78 & 1.24 & -1.87 & 0.844 \\
Masculino & 185 & 6.82 & 1.41 & & \\
& & & & & \\
Kinetics & & & & & \\
Feminino & 152 & 19.53 & 7.27 & 1.668 & 0.096 \\
Masculino & 214 & 18.31 & 6.66 & & \\
& & & & & \\
Mental & & & & & \\
Feminino & 152 & 19.92 & 7.36 & 2.181 & 0.030 \\
Msaculino & 214 & 18.29 & 6.85 & & \\
\hline
\end{tabular}

Para os cálculos relativos à variável eu-atleta foram excluídos os não praticantes.
Para as outras comparações mantemos todos os elementos da amostra. De seguida comparamos os grupos em função da modalidade praticada (individuais vs colectivos) para as mesmas variáveis dependentes.

Os resultados demonstraram não existir diferenças estatisticamente significativas entre ambas as preferências de modalidade praticada.

\section{Quadro III. Diferenças entre tipo de desporto para as variáveis kinésicas, mental e auto percepção.}

\begin{tabular}{|lccccc|}
\hline EU ATLETA & $\mathrm{N}$ & $\mathrm{M}$ & $\mathrm{DP}$ & $\mathrm{t}$ & $\mathrm{P}$. \\
\hline Individual & 143 & 6.84 & 1.37 & 361 & 0.718 \\
Colectivo & 117 & 6.78 & 1.34 & & \\
& & & & & \\
Kinetics & & & & & \\
Individual & 143 & 19.04 & 6.70 & 1.605 & 0.110 \\
Colectivo & 117 & 17.77 & 5.81 & & \\
& & & & & \\
Mental & & & & & \\
Individual & 143 & 18.96 & 7.08 & 1.688 & 0.093 \\
Colectivo & 117 & 17.58 & 5.89 & & \\
\hline
\end{tabular}

Também procuramos saber se existiam diferenças significativas entre os grupos em função da escolaridade. Dos cálculos realizados constatamos que não há diferenças estatisticamente significativas para a variável eu-atleta, mas que existiram paras as Kinetics $(t=2.766$. $p=0.0029$ e mental $(t=$ 2.057, $\mathrm{p}=0.019$ ).

Quando tomámos em consideração os anos de competição como variável independente, constatamos que apenas para a variável Eu-atleta se verificam diferenças significativas entre os grupos.

O teste post-hoc identificou a diferença entre os sujeitos do grupo 1 ( 0 a 5 ) e os do grupo 2 (6 a 10) $(F=9.658 . p=0.000)$. Os valores sugerem que os grupos 2 e 3 são muito semelhantes entre si. As comparações intra-grupos de escolaridade não evidenciaram diferenças estatisticamente significativas. 


\section{Quadro IV.Diferenças entre o nivel de ensino para as variáveis kinésicas, mental e auto percepção.}

\begin{tabular}{|lccccc|}
\hline EUATLETA & $\mathrm{N}$ & $\mathrm{M}$ & $\mathrm{DP}$ & $\mathrm{t}$ & $\mathrm{p}$. \\
\hline UTAD & 205 & 6.81 & 1.77 & .071 & 0.944 \\
12A & 55 & 6.80 & 1.90 & & \\
Kinetics & & & & & \\
UTAD & 205 & 19.03 & 6.54 & 2.766 & 0.002 \\
$12 A$ & 55 & 16.40 & 5.03 & & \\
Mental & & & & & \\
UTAD & 205 & 18.77 & 6.85 & 2.057 & 0.019 \\
12A & 55 & 16.52 & 5.32 & & \\
\hline
\end{tabular}

Quadro V. Diferenças por anos de prática para as variáveis kinésicas, mental e auto percepção.

\begin{tabular}{|lccccc|}
\hline EU ATLETA & N & M & DP & $F$ & $p$. \\
\hline 0 a 5 & 129 & 6.45 & 1.52 & 9.658 & 0.000 \\
6 a 10 & 117 & 7.17 & 1.09 & & \\
11 e + & 24 & 7.17 & 1.05 & & \\
Kinetics & & & & & \\
0 a 5 & 129 & 18.80 & 6.03 & 0.408 & 0.666 \\
6 a 10 & 117 & 18.25 & 6.34 & & \\
11 e + & 24 & 17.71 & 7.99 & & \\
Mental & & & & & \\
0 a 5 & 129 & 18.91 & 6.19 & 0.964 & 0.383 \\
6 a 10 & 117 & 17.99 & 6.79 & & \\
11 e + & 24 & 17.76 & 7.80 & & \\
\hline
\end{tabular}

\section{DISCUSSÃO E CONCLUSÕES}

Das várias hipóteses nulas elaboradas, para o propósito deste estudo, foram rejeitadas 4, nomeadamente quando comparámos os grupos em função dos sexos, nível de escolaridade e anos de prática. Relativamente às diferenças por sexo, constatámos que os indivíduos do sexo feminino apresentaram melhores valores que os do sexo masculino na componente mental da imagética. Quanto ao nível de escolaridade foram os mais avançados que obtiveram melhores indicadores, tanto para a componente kinética como para a mental. Quando assumimos os anos de prática como variável independente, verificámos que os indivíduos com mais anos de prática obtiveram melhores valores.

Estes resultados tendem a validar resultados anteriormente obtidos. Isaac e Marks (1994), levaram a cabo um conjunto de estudos de entre os quais realçamos os que mais se relacionam com o tipo de variáveis também por nós estudadas. Assim, estes estudiosos constaram que os estudantes de educação física, quando comparados com outros, tendiam a obter melhores resultados. O presente estudo corrobora esses dados. Tal como Isaac e Marks, sugerimos que estas diferenças resultam do facto de os estudantes de Educação Física possuírem um maior repertório motor que os outros. O mesmo argumento pode ser utilizado para argumentar as diferenças que encontrámos tanto para os níveis de ensino, como para os anos de prática desportiva dos grupos estudados. Este argumento poderá, ainda, ser reforçado se aceitarmos que os estudantes de Educação Física, para serem proficientes nas suas actividades, necessitam ser eficazes no planeamento e execução de sequências de comportamentos motores complexos, tal como estes são referentes aos seus próprios corpos.

Isaac e Marks também compararam atletas por nível de rendimento. Os seus resultados evidenciaram que os atletas de elite obtinham valores mais altos que os não-elite. Nesta situação, os autores demonstraram que quanto maior for a experiência desportiva maiores são os valores obtidos na variável vivacidade da imagética. A explicação dos resultados obtidos foi que para os atletas de elite a vivacidade da experiência apresenta-se como componente fundamental para o processo de aprendizagem e melhoria do rendimento motor e desportivo. Este tipo de argumento é validado pelos resultados por nós obtidos quando comparámos os atletas por anos de prática, uma vez que os atletas com mais anos de prática também são os que 
apresentaram melhores índices de rendimento desportivo.

Quando Isaac e Marks compararam praticantes de desportos colectivos com os de individuais, constataram que os últimos obtinham valores mais altos que os primeiros. No presente estudo não encontrámos diferenças estatisticamente significativas, porém as médias obtidas pelos praticantes de modalidades individuais apresentaram valores mais altos que os de desportos colectivos. Assim, se por um lado os valores do nosso estudo não validaram estaticamente os de Isaac e Marks, também foi verdade que a direcção dos resultados do presente estudo sugerem que, efectivamente, poderá existir essa tendência diferenciadora. Em parte, poderemos aceitar o argumento que ambos os estudos recorreram a instrumentos diferentes.

Se tomarmos com referência comparativa os valores médios obtidos no estudo realizado por Munroe, Hall, Simms e Weinberg (1998) constatamos que os praticantes de modalidades individuais não obtêm valores mais altos que os de desportos colectivos. À partida parece não existir coerência entre os resultados publicados nos diferentes estudos. No entanto, as diferenças apontaram para a necessidade de um maior rigor no desenho dos estudos, assim como para a descrição das metodologias seguidas, de forma a que seja possível duplicá-las.

As explicações que frequentemente encontrámos na literatura da psicologia do desporto apresentam várias limitações que resultam do facto de os psicólogos manifestarem o que Malina (Comunicação pessoal) classificou de biofobia. Assim, sugerimos que as explicações para as diferenças obtidas neste estudo têm de ser complementadas com os princípios propostos pelas teorias da aprendizagem motora.

A imagética mental, uma das formas de treino mental é um procedimento de evocação que pode produzir efeitos em vários locais do sistema motor Schmidt \& Wrisberg, 2000). A evocação mental pode envolver apenas aspectos de ordem cognitiva-simbólica, de tomada de decisão, de previsão de estratégias e acções possíveis de acontecer estimando os resultados prováveis para a situação real. Mas a imagética pode ser considerada de uma forma mais abrangente do que uma simples perspectiva de aprendizagem cognitiva-simbólica, como demonstraram os resultados obtidos na investigação realizada por Jacobson (1930, in Schmidt \& Wirsberg, 2000). Sempre que representamos mentalmente essa traduz-se em impulsos que podem ser mensurados na forma de actividade eléctrica nos músculos, que embora mínima, é suficiente para produzir feedback.

Tendo em conta estas duas perspectivas: (i)- teoria de aprendizagem simbólica e (ii)- teoria psico-neuro-muscular, como propostas por Zaichwosky e Fuchs (1988) a imagética mental pode ser benéfica quer para níveis iniciais de aprendizagem, quer para níveis mais avançados.

Inicialmente a teoria de aprendizagem simbólica defendeu que a prática mental reforça as componentes cognitivas da tarefa. Estas foram consideradas importantes para as primeiras fases de aprendizagem. Por esta razão foram tidas como mais eficazes para os atletas que se encontram em fase de iniciação. Esta posição alicerça-se proposta de Fitts e Posner (1967) que defende que o estádio inicial de aprendizagem é essencialmente de natureza cognitiva. Ainda de acordo com estes autores a imagética facilita a evocação das componentes cognitivas para criar uma representação mental (forte) da tarefa antes de o indivíduo as a executar.

Os proponentes da perspectiva psico-neuro-muscular demonstraram que existe uma ligação entre a mente e o movimento durante o período em que o indivíduo se submete à prática da imagética. Esta proposta foi confirmada, pela primeira vez, por Jacbson 
(1930), quando registou a actividade eléctrica dos músculos envolvidos na acção que os sujeitos estavam a "visualizar". Apesar dessa actividade ser mínima e de não se registar um movimento corporal perceptível a olho nu, a mensuração dos impulsos permitiu confirmar que existe um plano de acção que é enviado do sistema nervoso central para os músculos.

Os benefícios da imagética foram explicados mais recentemente por Mackay (1981, in Schmidt \& Wrisberg, 2000). De acordo com este autor, as unidades musculares envolvidas são instruídas para a acção durante a prática de imagética mental. A extensão dos benefícios deste treino mental depende da quantidade de prática anterior ou da experiência que $\mathrm{o}$ atleta tenha tido relativamente à realização da tarefa em causa. De acordo com esta perspectiva existe algo mais do que uma simples aprendizagem cognitiva dos elementos da tarefa, justificando desta forma porque razão os atletas de alto nível beneficiam consideravelmente mais da imagética mental do que os iniciados.

Durante a imagética mental os programas motores são enviados para os músculos, e, embora as contracções musculares sejam dificilmente visíveis, elas existem. São micro-contracções suficientes para que os corpúsculos de Golgi, órgãos muito sensíveis a pequenas forças, enviem feedback da acção executada.

O feedback interno é considerado um elemento fundamental no controlo dos movimentos, tal como proposto nas teorias de Adams (1971) e de Schmidt 1975). O conteúdo informativo do feedback intrínseco permite comparar o valor visado com a resposta produzida. Quando se verifica uma discrepância entre os dois, regista-se um erro que o sistema vai tentar corrigir (função de correcção). Mas se o objectivo foi cumprido a informação de retorno (feedback) confirma a acção como adequada (função de reforço). A informação sobre o erro é fundamental para a aprendi- zagem, tal como preconizam as teorias de Adams (1971) e de Schmidt (1975). O valor do feedback (correcção ou reforço) depende da capacidade de processamento de informação do sujeito, variando de acordo com a fase de aprendizagem e com a idade do indivíduo. Em parte este argumento poderá, de alguma forma pode explicar porque há uma tendência para que os indivíduos mudarem de uma modalidade de imagética para a outra.

Os resultados superiores obtidos pelos atletas que praticam desportos individuais, quando comparados com os que praticam desportos colectivos podem ser interpretados tendo em conta os resultados obtidos por Feltz e landers (1983). Os autores verificaram que, quando não foi tomado em consideração o nível de prestação, o treino mental foi mais eficaz para as tarefas com um maior número de componentes simbólico-cognitivas de prestação. Este facto, pode de alguma forma explicar o porquê de resultados mais positivos nos atletas de desportos individuais. Nos desportos individuais a incerteza quer a nível espacial, quer temporal, quer de ocorrência das acções é menor do que nos desportos colectivos, dado que um maior número de jogadores torna mais imprevisível o número de acções motoras possíveis de serem executadas. Desta forma, o aspecto simbólico-cognitivo assume um papel preponderante no processo de concentração, da execução da estratégia e táctica relacionada com a seuência das tarefas a desempenhar a nível das tomadas de decisão e de antecipação de estratégias e acções possíveis de acontecer quando se estimam os resultados prováveis para a situação real.

Apesar de não se terem registado diferenças significativas entre rapazes e raparigas, estas apresentaram melhores resultados. Estes dados podem ser interpretados com base nas propostas da teoria de esquema de Schmidt (1975). De acordo com esta teoria as condições de prática variada levam ao fortalecimento de esquemas 
de resposta mais genéricos, robustos e plásticos favorecendo a sua adaptabilidade a múltiplas situações. As alterações das condições de realização das tarefas vai ter como consequência que o indivíduo retenha em memória diferentes e variados parâmetros de especificação da resposta como também diversificadas consequências sensoriais recebidas assim como valores efectivos de resposta que produzem efeitos positivos na retenção e transfer de aprendizagem.

Tendo por base os estudos realizados no âmbito desta teoria como os de Shapiro e Schmidt (1982) e os de Wrisberg e Ragsdale (1979) verificou-se que a prática variada foi mais eficaz em crianças e em adultos do sexo feminino do que a constante. Estes resultados foram explicados tendo em conta o pressuposto de que as crianças e o sexo feminino apresentaram um menor número de vivências motoras, são por isso mais "younger" in a movement sense", como defende Schmidt e Lee (1999), pelo que os seus esquemas motores estão ainda em formação sendo por isso mais sensíveis à prática. A prática variada produz uma regra (esquema) que é formada pela relação estabelecida entre todos os resultados obtidos em experiências anteriores e os valores dos parâmetros utilizados para produzir as respostas. Esta esquema é registado em memória e pode ser utilizado para seleccionar um novo conjunto de parâmetros para a realização de um próximo movimento. Com a prática variada a teoria prediz que a aprendizagem de regras é mais eficaz com uma prática variada do que com uma prática constante.

De acordo com esta perspectiva, a imagética mental pode ser considerada nesta perspectiva, como uma prática variada na tarefa, dado que, como confirma a teoria psiconeuro-muscular existe contracção muscular, os programas motores são projectados e enviados para os músculos eo indivíduo recebe feedback. De acordo com a mesma teoria, todas estas informações são essenciais para a formação dos esquemas motores responsáveis pela realização do movimento.

Notas finais. A investigação científica na área da psicologia diferencia-se das outras ciências sociais porque tem como objectivo identificar como os indivíduos se diferenciam entre si. Neste estudo procuramos diferenciar jovens praticantes de desportos individuais e colectivos os resultados evidenciam algumas diferenças estatisticamente significativas. Da discussão que levamos a cabo realça-se o facto de ser necessário integrar outras variáveis que vão para além das tradicionalmente tomadas em consideração na análise dos psicólogos, que tendem a ignorar algumas componentes, como é exemplo a experiência motora. Tomando em consideração os argumentos apresentados, julgamos importante levar a cabo um estudo que tenha por objectivo comparar jovens com prática e sem prática desportiva. $\mathrm{Na}$ eventualidade da nossa teorização estar correcta, não será surpresa que os indivíduos sem prática desportiva, quando sujeitos ao mesmo protocolo experimental implementado neste, evidenciem níveis superiores de imagética cinética e provavelmente mental. A proposta teórica é que a execução das tarefas a que são sujeitos, por se constituírem como estímulos novidade tenderão a ser percepcionados de uma forma mais acentuada e, consequentemente, projectados em representação numérica superior à reportada pelos indivíduos com mais experiência motora.

\section{BIBLIOGRAFIA}

ADAMS, J.A.(1971). A closed-loop theory of motor learning. Journal of Motor Behavior, 3.11-150.

ANGELL, j. (1912). Methods for the determination of mental imagery. Psychologícal Monographs. 13, 61-108.

BETTS, G. M. (1909). The distribution and function of mental imagery. New York: Teacher College, Columbia University. 
BONE, P. F., \& ELLEN, P. S. (1992). The generation and consequences of communication evoked imagery. Journal of Consumer Research, 19, 93-104.

BUTTS, N., GUSHIKEN, T., \& ZARINUS, B. (1985). The elite athlete, Champaign, I I I.: Life Enhancement Publications.

CLARK, L. V. (1960). Effect of mental practice on the development of a certain motor skill. Research Quarter/y. 31, 360-369.

CLARK, LV. (1960). The effect of mental practice an the development of a certain motor skill. Research Quarterly, 31, 560-569.

COLVIN, S.S. (1908). The nature ofthe mental image. Psychological Review, 15, 355-364.

CORBIN, C. B. (1967). The effects of covert rehearsal on the development of a complex motor skill. Journal of General Psychology, 76, 143-150.

CROOKENTON, I. (1982). Errors in tennis. Trabalho apresentado na National Sports Coaches Conference, Hamilton, Nova Zelândia.

CSIKSZENTMIHALYI, M. (1990). Flow: The psychology of optimal experience. New York: Cambridge University Press.

CULLINAN, B. (1999). Imagery is of the essence: Teaching children visualization and abstract thinking through poetry. Instructor, 108, 45-49.

FECHNER, G.T.. (1860). Elements of psychophysics (Traduzido por H.E. Adler) New York: Rinehart \& Winston.

FELTZ, D. L., LANDERS, D. M. \& BECKER, B. B. (1988). A revised meta-analysis of the mental practice literature on motor skill learning. In D. Druckman \& J. Swets (Eds.) Enhancing human performance: Issues, theories, and techniques. The National Academy Press.

FELTZ, D.C., \& LANDERS, D.M. (1983). The effects of mental practice on motor skill learning and performance: A meta-analysis. Journal of Sport Psychology, 5, 25-57.

FERNALD, M. L. (1912). The diagnosis of mental imagery. Psychological Monographs, 14,1-9.

FITTS, P. M., \& POSNER M. J. (1967). Human performance. Belmont, CA: Brooks/cole.

GALTON, F. (1880). Statistics of mental imagery. Mind, 19, 303-318.

GARFIELD, C. (1985). Peak Performance: Mental Training Techniques of the World's Greatest Athletes. Los Angeles: Warner Brothers.

GORDON, S., WEINBERG, R., \& JACKSON, A (1994). Effect of internal and external imagery on cricket performance. Journal of Sport Behavior, 17,60-76.

GOULD, D. , TAMMEN, V., \& MURPHY, S.M. (1989). An examination of the u.s. Olympic sport psychology consultants and the services they provide. The Sport Psychologist, 5, 300-312.

HALE, BD. (1982). The effects of internal and external imagery on muscular and ocular concomitant. Journal of Sport Psychology, 4, 379-387.

HALL, C. R., RODGERS, W. M., \& BARR, K. $A$ (1990). The use of imagery by athletes in selected sports. The Sport Psychologist, 4, 1-10.

HALL, C., SCHMIDT, D., DURAND, M., \& BUCKOLZ, E. (1994). Imagery and motor skills acquisition. In A.A. Sheikh \& E.R Korn (Eds.), Imagery in sports and physical performance (pp. 121-134). Amityville, NY: Baywood Publishing Company.

HALL, G., \& PONGRAC, J. (1971). Movement imagery: Questionnaire. University of Western Ontario, London, Ontario.

HALL, C., MACK, D.E., PAIVIO, A., \& HAUSENBLAS, H. (1998). Imagery use by athletes: Development of the Sport Imagery Questionnaire. Internationol Journal of Sport Psychology, 29, 73-89. 
HARRIS, D. V., \& HARRIS, B., (1987). Psicologia del deporte, Nova York: Leisure Press.

HORN, T.(1992). Advances in Sport Psychology, Champaign, III.:Human Kinetics.

ISAAC, A., \& MARKS, D. (1994). Individual differences in mental imagery experience: Developmental changes and specialization . British Journal of Psychology, 85, 479-500.

JACOBSON, E. (1930). Electrophysiology of mental activities. American Journal of Physiology, 94, 22-34.

JANSSEN, A, \& SHEIKH, A (1994). Enhancing athletic peformance through imagery: An overview. In Anees A.Sheikh and Errol R. Korn (1994) Imagery in sports and physical performance. New York: Baywood Pub..

JOWDY, D. P., MURPHY, S. M. \& DURTSCHI, S. (1989). An assessment of the use of imagery by elite athletes: Athlete, coach, and psychologist perspectives. (report). Colorado Springs, Co: United States Olympic Committee.

KOSSL YN, S. (1980). Image and mind. Cambridg. MA: Harvard Univ. Press.

KOSSLYN, S. (1988). Aspects of a cognitive neuroscience of mental imagery. Science, 240,1621-1627.

LENNINGS, C. J. (1996). Adolescent aggression and imagery: Contribution from object relations and social cognitive theory. Adolescence, 31, 831-841.

MAHONEY, M.J., \& AVENER, M. (1977). Psychology of the Elite Athlete: An Exploratory Study. Cognitive Therapy and Research, 1, 135-141.

MARTENS, R. (1987) Coaches guide to sport psychology, Champaign, III.: Human Kinetics.
MORRIS , T., \& SUMMERS, J. (1995). Sport psychology: Theory, applications and issues. New York: JohnWilley \& Sons.

MUNROE, K., HALL, C., SIMMS, S., \& WEINBERG, R. (1998). The influence of type of sport and time of season on athlete's use of imagery. The Sport Psychologist, 12, 440-449.

MURPHY.S.M.,JOWDY, D.P., \& DURTSCHI, S.K. (1990). Report on the united states Olympic committee survey on imagery use in sports. Colorado Springs: US Olympic Training Center.

NIDEFFER, R. (1985). Athlete's guide to mental training, Champaign, III.: Human Kinetics.

O'HALLORAN, T. (1985). The role of preferred cognitive style in lhe effectiveness of imagética training. International Journal of Sport Psychology, 25, 19-31.

O'HALLORAN, A. M., \& GAUVIN, L. (1994). The role of preferred cognitive style in the effectiveness of imagery training. Int. J. Sport Psychology, 25, 19-31.

ORLICK, T. (1986). Psyching for sport, Champaign, III.: Leisure Press.

PAVIO, A. (1985). Cognitive and motivational functions of imagery in human performance. Canadian Journal of Applied Sport Sciences, 10, 22-28.

PERRY, C., \& MORRIS, T. (1995) Mental Imagética in Sports. In Tony Morris e Jeff Summers (Eds.) Sport Psychology: Theory, Applications and Issues. New York: John Willey and Sons.

RICHARDSON, A (1967a). Mental Practice: A Review and discussion. Part I. Research Quarterly, 38, 95-107.

RICHARDSON, A (19678). Mental practice: A review and discussion. Part 11. Research Quarter/y, 38, 263-273.

ROTELLA, R.J., GANSNEDER, B., OJALA, D., \& BILLING, J. (1980). Cognitions and coping strategies of elite skiers: An 
exploratory study of young developing athletes. Journal of Sport Psychology, 2, 350-354.

SCHAUB,A (1911). On the intensity of images. American Journal of Psychology, 22, 346-368.

SCHMIDT, R. A (1982). Motorcontrol learning: A Behavioral Emphasis. Champaign, III.: Human Kinetics.

SCHMIDT, R. A(1975). A schema theory of discrete motor skill learning. Psychological Review, 82, 225-260.

SCHMIDT, R, \& LEE, T. (1999). Motor Control And Learning. A Behavioral Emphasis. (3a Ed.). Champaign III: Human Kinetics.

SCHMIDT, R.A., \& WRISBERG, C. A. (2000). Motor Learning and performance. A Problem-Based Learning Approach. ( $2^{\mathrm{a}}$ Ed.). Champaign III: Human Kinetics.

SHAPIRO, D. C., \& SCHMIDT, R. A. (1982). The schema theory: recent evidence and developmental implications. In J.AS. Kelso \& J.E. Clark (Eds.), The development of movement control and co-ordination (pp.113150). New York: Wiley.

START, K.B. (1962). The influence of subjectivity assessed "games ability" on gain in motor performance after practice. Journal of General Psychology, 67, 159-173.

STETSON, RM. (1896). Types of imagination. Psychological Review, 3, 398-410.

SUINN, R. (19894). Visualization in sport. In A. A Sheikh and E. R Korn (Eds.) Imagery in sport and physical performance (pp. 23-44). Amityville, NY: Baywood pub Camp.

SWARTZ, D, (1982). Visualização interior, NWS, IV, 24, Nov/Dez.

SYER, J., \& CONNOLLY, C., (1987). Sporting body, sporting mind, Englewood Cliffs, N.J.: Prentice Hall

TUSEK, D., CHURCH, J. M., \& FAZIO, V. W. (1997). Guided imagery as a coping strategy for perioperative patients. AORN JOURNAL, $66,644-649$.

UNGERLEIDER, S., \& GOLDING, J. M. (1991). Mental practice among Olympic athletes. Perceptual and MotorSkills, 72, 1007-1017.

VACCARO, K.C. (1997). Teaching strategies: The application of found images in dance and sport. JOPERD. The Journal of Physical Education, Recreation \& Dance. 68, 45-50.

VASCONCELOS-RAPOSO, J. (1994). Os factores psico-socio-culturais que influenciam e determinam a busca da excelência pelos atletas de elite portgueses. Dissertação de Doutoramento, defendida na Universidade de Trás-os-Montes e Alto Douro.

VASCONCELOS-RAPOSO, J. (1994A). Manual de Treino de definição de Objectivos. Vila Real; UTAD/ FPN.

VASCONCELOS-RAPOSO, J., \& COSTA, G. (1997). Caracterização da prática da visualização em estudantes de Educação Física. Vila Real: UTAD-SDE.

VEALEY, R, (1986). Imagética Training for Performance Enhancement. In Jean Williams (Ed.) Applied sport psychology: Personal growth to peak performance. Mountain View, CA: Mayfield Publishing Comp..

VEALEY.R.E., WALTER, S.M. (1993). Imagery training for performance enhancement and personal development. "In Jean Williams (Ed.) Applied sport psychology: Personal growth to peak performance. Mountain View, CA: Mayfield Publishing Comp..

WASHBURN, M. F. (1916). Mental imagery. Boston: Houghton.

WEINBERG, R. (1982). The relationship between mental preparation strategies and motor performance: A review and critique. Quest, 33,190-213.

WEINBERG, R., \& RICHARDSON, P. (1990). Psychology of officiating, Champaign, III.: Human Kinetics. 
WEINBERG, R, (1988). The mental advantage, Champaign, III.: Leisure Press.

WIGGINS, D. K. (1984). The history of sport psychology in North America. In J. M. Silva and R. Weinberg, Psychological foundation of sport. Champaign, III: Human Kinetics.

WOOD, G. (1977). Fundamental of psychological research. Boston: Little, Brown and Comp.
WRISBERG, CA, \& RAGSDALE, M.R (1979). Further tests of Schmidt's schema theory: Development of a schema rule for a coincident timing task. Journal of Motor Behavior, 11, 159-166.

ZAICHKOWSKY, L., \& FUCHS, C.Z. (1988). Biofeedback applications in exercise and athletic performance. Exercise \& Sport Science Review, 16, 381-419.and Spor ScienceReview, 16, 381-419. 